\title{
PREST-plus identifies pedigree errors and cryptic relatedness in the GAW18 sample using genome-wide SNP data
}

\author{
Lei Sun ${ }^{1,2}$, Apostolos Dimitromanolakis $3^{3^{*}}$ \\ From Genetic Analysis Workshop 18 \\ Stevenson, WA, USA. 13-17 October 2012
}

\begin{abstract}
Pedigree errors and cryptic relatedness often appear in families or population samples collected for genetic studies. If not identified, these issues can lead to either increased false negatives or false positives in both linkage and association analyses. To identify pedigree errors and cryptic relatedness among individuals from the 20 San Antonio Family Studies (SAFS) families and cryptic relatedness among the 157 putatively unrelated individuals, we apply PREST-plus to the genome-wide single-nucleotide polymorphism (SNP) data and analyze estimated identity-by-descent (IBD) distributions for all pairs of genotyped individuals. Based on the given pedigrees alone, PREST-plus identifies the following putative pairs: 1091 full-sib, 162 half-sib, 360 grandparent-grandchild, 2269 avuncular, 2717 first cousin, 402 half-avuncular, 559 half-first cousin, 2 half-sib+first cousin, 957 parent-offspring and 440,546 unrelated. Using the genotype data, PREST-plus detects 7 mis-specified relative pairs, with their IBD estimates clearly deviating from the null expectations, and it identifies 4 cryptic related pairs involving 7 individuals from 6 families.
\end{abstract}

\section{Background}

Mis-specified pedigree relationship and cryptic relatedness often occur in family and population data. The potential causes of such errors are numerous, including undocumented nonpaternity, nonmaternity, adoption, mating between relatives, sample duplication or swap. It is well known that such errors, if undetected, can affect the accuracy or power of both linkage and association studies, as well as have adverse effects on other aspects of the analyses such as population stratification [1-6].

Genome-wide marker data can provide accurate information on the genetic relatedness among individuals. For linkage scans, a number of statistical methods have been proposed and implemented, including RELCHECK [1], RELATIVE [7], PEDCHECK [8], SIBERROR [9], PREST [10,11], GRR [12], and ECLIPSE [13], among others. More recently, PLINK [14] and PREST-plus [5,6,15] have been developed for analysing the high-throughput SNP data

\footnotetext{
* Correspondence: apostol@cs.toronto.edu

${ }^{3}$ Department of Clinical Biochemistry, University Health Network, Canada
} Full list of author information is available at the end of the article collected from genome-wide association studies (GWAS) or next-generation sequencing (NGS) experiments.

There are 3 main differences between PREST-plus and PLINK. First, PREST-plus uses the maximum likelihoodbased IBD estimation method, which has more statistical power, whereas PLINK relies on the method-of-moments approach, which is computationally more efficient. Second, PREST-plus identifies both pedigree errors and cryptic relatedness in linkage or association studies with family data, population sample, or both, whereas PLINK is primarily suitable for detecting cryptic relatedness in GWAS with population sample. Third, PREST-plus provides a formal hypothesis testing framework, which can be useful when a potential error has been being identified, whereas PLINK provides point IBD estimation only $[5,6]$. The Genetic Analysis Workshop 18 (GAW18) data, similar to many emerging large-scale GWAS and NGS studies, include multigeneration large pedigrees. The genetic relationships between individuals in such data are not limited to simple types such as parent-offspring or siblings. Consequently, we focus here on the methodology 
implemented in PREST-plus $[5,6,15]$ and discuss PLINK [14] when relevant.

\section{Methods}

\section{Relationship estimation and testing}

The relatedness between a pair of individuals can be summarized by the IBD probability distribution, $p=\left(p_{0}, p_{1}, p_{2}\right)$. It describes the probability of a randomly sampled marker to have 0,1 , or 2 common ancestry alleles between 2 individuals. Using the available genotype data, PREST-plus estimates the most likely IBD distribution by obtaining $\hat{p}=\left(\hat{p}_{0}, \hat{p}_{1}, \hat{p}_{2}\right)$ that maximizes the quantity

$$
L(p)=\sum_{m=1}^{M} \log \left(P\left(G_{m} ; p\right)\right)=\sum_{m=1}^{M} \log \left(\sum_{i=0,1,2} P\left(G_{m} \mid D_{m}=i\right) p_{i}\right),
$$

where $G_{m}$ is the genotype at marker $m$ and $D_{m}=i$ denotes the number of alleles shared IBD by the pair at that marker. The maximization is efficiently achieved by an application of the expectation-maximization (EM) algorithm $[10,16,17]$. In contrast, PLINK [14] estimates the IBD distribution using the method-of-moments approach, which is less powerful than the likelihood-based method.

Estimation of IBD distribution can often provide sufficient information to identify pedigree errors and cryptic relatedness $[5,6]$. However, it is useful to provide statistical evidence beyond point estimation. To this end, we apply the maximum likelihood ratio test (MLRT) to formally evaluate whether the observed genotypes $G$ are compatible with the null putative relationship type, $R_{O}[10]$. Briefly, $M L R T=\log (\hat{L}(A))-\log \left(L\left(R_{0}\right)\right)$, where $L\left(R_{0}\right)$ is the likelihood calculated for the hypothesized $R_{0}$, and $\hat{L}(A))=\max \left\{L\left(R_{1}\right)\right\}, R_{1} \in A$, is the maximum likelihood calculated over a set of alternatives as given in Table 1. Statistical significance of $M L R T$ is then assessed using simulation, because 2 "MLRT does not follow the usual Chisq distribution [10]. Efficient implementation of the test to high-throughput genotype data requires pruning of the SNPs so that they are not in linkage disequilibrium (LD) $[5,6]$.

\section{Data analyses}

The GWAS data of GAW18 consist of 959 individuals genotyped at 472,049 SNPs. Among the 959 individuals, 4 are removed for low genotyping rate (plink -MIND $>0.8$ ), and 141 individuals are in the "UNREL.txt" file that contains the maximum set of putatively unrelated individuals. Among the 472,049 SNPs, 50,000 remain after minor allele frequency (MAF) and LD pruning (plink-indep-pairwise 200500.2 -maf 0.05). We then conduct 3 sets of analyses, with analyses 1 and 2 focusing on relationship estimation and analysis 3 performing hypothesis testing.
Analysis 1 detects pedigree errors and cryptic relatedness in the 955 genotyped individuals from the 20 SAFS families using PREST-plus (prest -geno datafamily.ped -map datafamily.map -wped-aped). It estimates the IBD distribution for any pair of individuals within a pedigree (-wped), as well as for any pair of individuals across pedigrees (-aped).

Analysis 2 detects cryptic relatedness in the 141 putatively unrelated individuals, using both PREST-plus (prest -geno dataunrel.ped -map dataunrel.map) and PLINK (plink -file plink -genome).

Analysis 3 performs formal hypothesis testing on the problematic pairs identified in analyses 1 and 2 . For computational efficiency, we first randomly select $\sim 2000$ SNPs from the set of $\sim 50,000$ SNPs. We then obtain the base pair (bp) physical map of the SNPs using build 36 coordinates and their corresponding centimorgan (cM) genetic map using the Rutgers combined linkage-physical map and linear interpolation. Finally, we perform the MLRT test as implemented in PREST-plus (prest-file data2k.ped -map data2k.map -pair fID1 indID1 fID2 indID2 - mlrt -c).

\section{Results}

Analysis 1: Relationship estimation within and across the 20 SAFS families

PREST-plus identifies 455,535 pairs of genotyped individuals, with the total number of genotyped SNPs (commark) ranging from 31,120 to 49,020 . Most of the 455,535 pairs have the putative relationship types considered by PREST-plus (see Table 1). (See Figure 1 of reference [5] for graphical illustrations of these relationship types.)

Figure 1 shows the estimated IBD distributions, $\hat{p}_{1}$ vs $\hat{p}_{0}$, stratified by the putative relationship, $R_{0}$, with the red cross marking the the IBD distribution expected for $R_{0}$. We observe a substantial number of pairs with their IBD estimates deviating from the null expectations. Table 2 provides detailed information for 7 clear outliers, indicating mis-specified relationships. For example, 2 putative half-sib pairs have close to $(0.25,0.5,0.25)$ of full-sib, while 1 putative avuncular pair has close to $(1,0,0)$ of unrelated. Analysis 3 below is to determine the statistical significance of the apparent deviation in the IBD estimates.

\section{Analysis 2: Relationship estimation among individuals in the "UNREL.txt" file}

In this analysis, there are $141 * 140 / 2=9780$ putatively unrelated pairs and the commark ranges from 32,280 to 49,010. Figure 2 displays $\hat{p}_{1}$ vs $\hat{p}_{0}$ for these pairs based on PREST-plus (left) and PLINK (right). Results clearly demonstrate the statistical efficiency of PREST-plus with overall less variation in the IBD estimates as compared to PLINK: 9338 pairs with PREST $\hat{p}_{0} \geq 0.98$ vs. 6538 pairs with PLINK $\hat{p}_{0} \geq 0.98$. For the 4 potential cryptic 
Table 1 IBD distribution $p=\left(p_{0}, p_{1}, p_{2}\right)$ and kinship coefficient $\phi=p_{1} / 4+p_{2} / 2$ for the relationship types (reltype) considered by PREST-plus

\begin{tabular}{clcccc}
\hline reltype coding in PREST-plus & \multicolumn{1}{c}{$\begin{array}{c}\text { Relationship type } \\
\text { (abbreviation) }\end{array}$} & \multicolumn{2}{c}{ Distribution of IBD sharing } & \multicolumn{2}{c}{ Kinship coefficient, $\phi$} \\
\cline { 3 - 5 } & & $\boldsymbol{p}_{\boldsymbol{0}}$ & $\boldsymbol{p}_{\boldsymbol{1}}$ & $\boldsymbol{p}_{\boldsymbol{2}}$ \\
\hline 11 & MZ-twin (MZ) & 0.000 & 0.000 & 1.000 & 0.50000 \\
10 & parent-offspring (PO) & 0.000 & 1.000 & 0.000 & 0.25000 \\
1 & full-sib (FS) & 0.250 & 0.500 & 0.250 & 0.25000 \\
9 & half-sib+first cousin (HSFC) & 0.375 & 0.500 & 0.125 & 0.18750 \\
2 & half-sib (HS) & 0.500 & 0.500 & 0.000 & 0.12500 \\
3 & grandparent-grandchild (GPC) & 0.500 & 0.500 & 0.000 & 0.12500 \\
4 & avuncular (AV) & 0.500 & 0.500 & 0.000 & 0.12500 \\
5 & first cousin (FC) & 0.750 & 0.250 & 0.000 & 0.06250 \\
7 & half-avuncular (HAV) & 0.750 & 0.250 & 0.000 & 0.06250 \\
8 & half-first cousin (HFC) & 0.875 & 0.125 & 0.000 & 0.03125 \\
6 & unrelated (UN) & 1.000 & 0.000 & 0.000 & NA \\
99 & other types (Others) & NA & NA & NA & NA \\
\hline
\end{tabular}

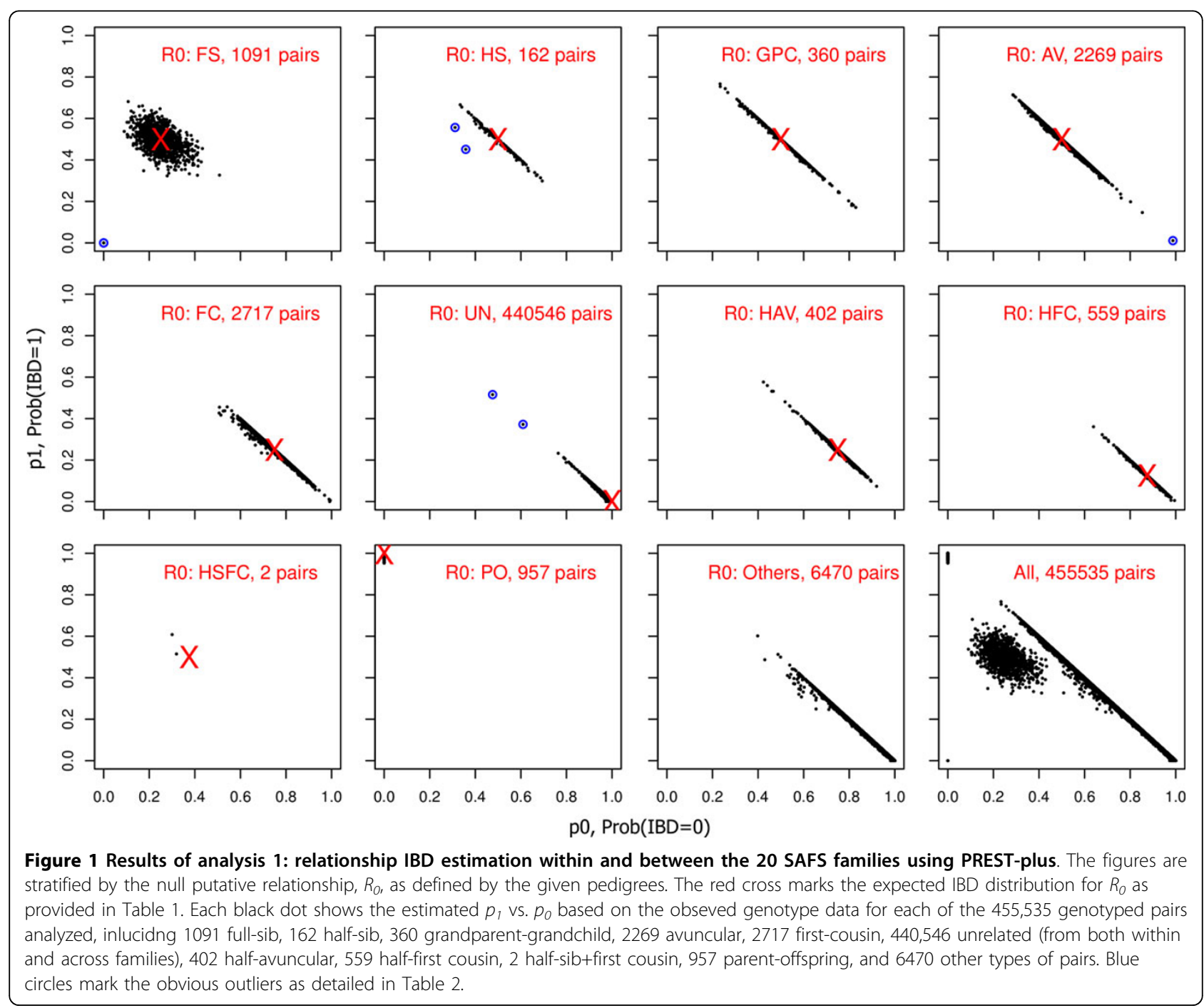


Table 2 Relationship estimation results for clear outliers in Figure 1 identified by analysis 1

\begin{tabular}{|c|c|c|c|c|c|c|c|c|}
\hline \multirow[b]{2}{*}{ FID $1^{a}$} & \multirow[b]{2}{*}{$I I D 1^{b}$} & \multirow[b]{2}{*}{ FID2 $^{a}$} & \multirow[b]{2}{*}{$\operatorname{IID} 2^{b}$} & \multirow[b]{2}{*}{ reltype $^{c}$} & \multirow[b]{2}{*}{ commark $^{d}$} & \multicolumn{3}{|c|}{ Estimated } \\
\hline & & & & & & $p_{o}$ & $p_{1}$ & $p_{2}$ \\
\hline 3 & T2DG0300174 & 3 & T2DG0300175 & 1 & 49009 & 0.0000 & 0.0000 & 1.0000 \\
\hline 4 & T2DG0400281 & 4 & T2DG0400282 & 1 & 48996 & 0.0000 & 0.0000 & 1.0000 \\
\hline 4 & T2DG0400265 & 4 & T2DG0400266 & 2 & 48994 & 0.358 & 0.4511 & 0.1909 \\
\hline 21 & T2DG2100946 & 21 & T2DG2100947 & 2 & 48957 & 0.3112 & 0.5566 & 0.1322 \\
\hline 21 & T2DG2100952 & 21 & T2DG2100966 & 4 & 48949 & 0.9876 & 0.0109 & 0.0015 \\
\hline 4 & T2DG0400207 & 4 & T2DG0400260 & 6 & 48955 & 0.4759 & 0.5157 & 0.0084 \\
\hline 4 & T2DG0400207 & 4 & T2DG0400247 & 6 & 47503 & 0.6094 & 0.3723 & 0.0182 \\
\hline
\end{tabular}

These 7 pairs of individuals have their estimated IBD distributions clearly deviating from the null expected values as specified in Table 1.

a Family ID.

b Individual ID.

c Relationship type as in Table 1.

$\mathrm{d}$ The number of common markers genotyped for both individuals.

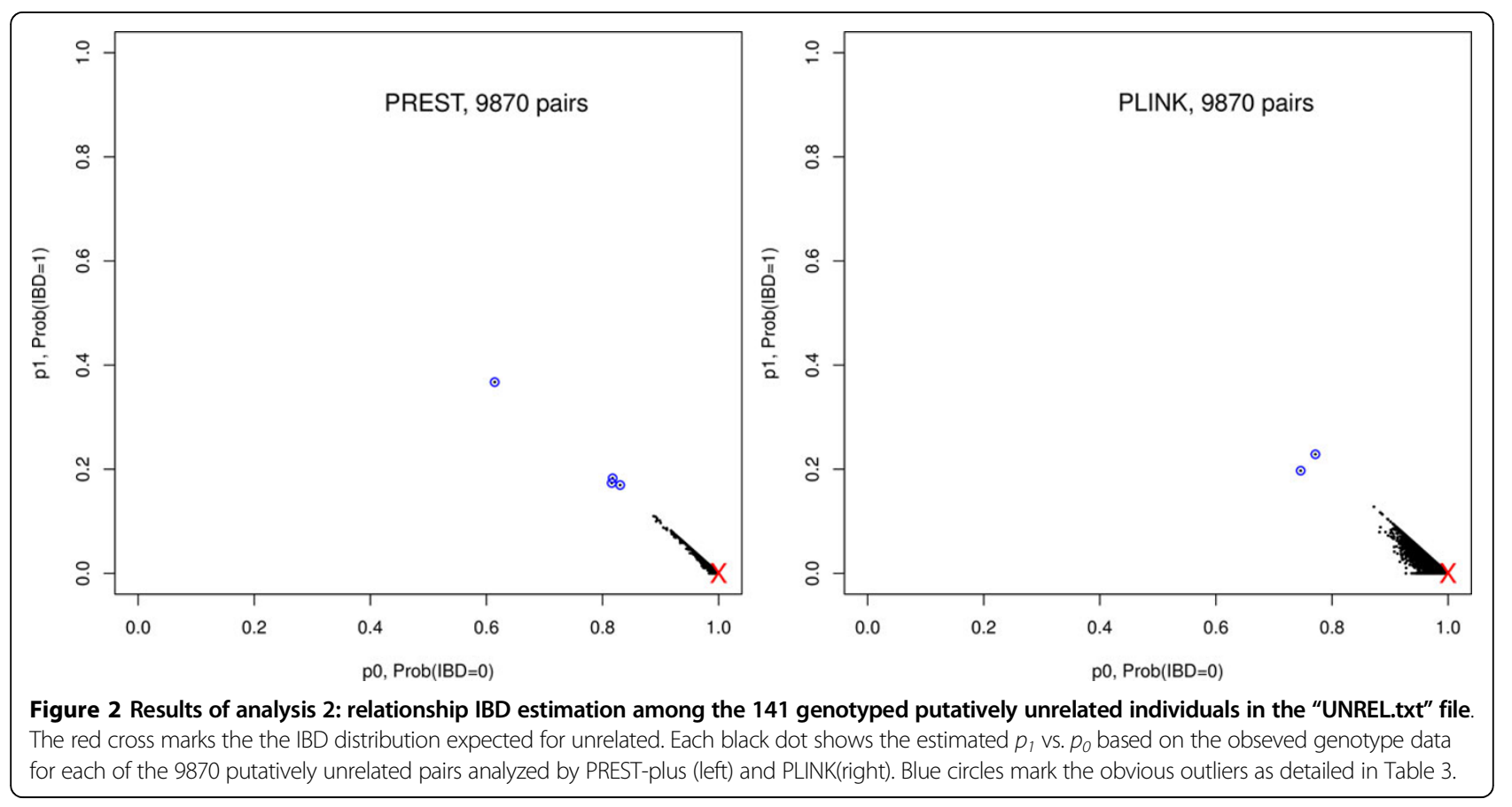

relateded pairs, the PREST-plus IBD estimates provide a better identification of the outliers (Table 3 ).

\section{Analysis 3: Relationship hypothesis testing of problematic pairs}

As a proof of principle, we focus on the 7 clear outliers identified in analysis 1 and the 4 pairs identified in analysis 2 (Table 4). Among the putatively unrelated pairs, the possible alternative relationship types range from half-first cousin to avuncular.

\section{Discussion}

The GAW18 "pedigree information was [previously] verified by estimated kinship coefficients, principal component analysis (PCA), and number of mendelian errors between parent and offspring samples." However, no other details are provided and our analyses show that pedigree errors and cryptic relatedness exist in the data.

The results presented here are based on the $\sim 50,000$ GWAS SNPs that have MAF greater than $5 \%$ and pair-wise LD less than 0.2. Our experience with PREST-plus shows that there is little improvement in estimation accuracy, once more than $\sim 50,000$ SNPs were used (typically with MAF $>5 \%$ ). Additional analyses with denser sets of SNPs confirmed this (results not shown). However, substantially more SNPs are needed for PLINK to achieve similar estimation 
Table 3 Relationship estimation results for clear outliers in Figure 2 identified by analysis 2

\begin{tabular}{|c|c|c|c|c|c|c|c|c|c|c|c|}
\hline \multirow[b]{2}{*}{ FID1 } & \multirow[b]{2}{*}{ IID1 } & \multirow[b]{2}{*}{ FID2 } & \multirow[b]{2}{*}{ IID2 } & \multirow[b]{2}{*}{ reltype } & \multirow[b]{2}{*}{ commark } & \multicolumn{3}{|c|}{ PREST-plus estimated } & \multicolumn{3}{|c|}{ PLINK estimated } \\
\hline & & & & & & $p_{o}$ & $p_{1}$ & $p_{2}$ & $p_{o}$ & $p_{1}$ & $p_{2}$ \\
\hline 9 & T2DG0901244 & 10 & T2DG1000566 & 6 & 48912 & 0.8159 & 0.1735 & 0.0105 & 1.0000 & 0.0000 & 0.0000 \\
\hline 8 & T2DG0800497 & 9 & T2DG0901244 & 6 & 48957 & 0.8304 & 0.1696 & 0.0000 & 1.0000 & 0.0000 & 0.0000 \\
\hline 21 & T2DG2100951 & 25 & T2DG2501033 & 6 & 48940 & 0.8174 & 0.1826 & 0.0000 & 0.7713 & 0.2287 & 0.0000 \\
\hline 4 & T2DG0400207 & 4 & T2DG0400247 & 6 & 47503 & 0.6142 & 0.3673 & 0.0185 & 0.7460 & 0.1972 & 0.0568 \\
\hline
\end{tabular}

These four pairs of individuals have their estimated IBD distributions clearly deviating from the values expected for unrelated pairs.

Table 4 Relationship testing results for clear outliers in Figure 1 identified by analysis 1, and in Figure 2 identified by analysis 2

\begin{tabular}{|c|c|c|c|c|c|c|c|c|c|}
\hline FID1 & IID1 & FID2 & IID2 & \multicolumn{2}{|c|}{ null reltype } & $p$ value & \multicolumn{2}{|r|}{ plausible reltype } & $p$ value \\
\hline \multicolumn{10}{|c|}{ The 7 outliers identified by analysis 1} \\
\hline 3 & T2DG0300174 & 3 & T2DG0300175 & 1 & full-sib & 0 & 11 & MZ-twins & N/A \\
\hline 4 & T2DG0400281 & 4 & T2DG0400282 & 1 & full-sib & 0 & 11 & MZ-twins & N/A \\
\hline 4 & T2DG0400265 & 4 & T2DG0400266 & 2 & half-sib & 0 & 9 & half-sib+first cousin & 0.254 \\
\hline 21 & T2DG2100946 & 21 & T2DG2100947 & 2 & half-sib & 0 & 9 & half-sib+first cousin & 0.432 \\
\hline 21 & T2DG2100952 & 21 & T2DG2100966 & 4 & avunuclar & 0 & 6 & unrelated & 0.891 \\
\hline 4 & T2DG0400207 & 4 & T2DG0400260 & 6 & unrelated & 0 & 2 & half-sib & 0.328 \\
\hline 4 & T2DG0400207 & 4 & T2DG0400247 & 6 & unrelated & 0 & 5 & first cousin & 0.752 \\
\hline \multicolumn{10}{|c|}{ The 4 outliers identified by analysis 2} \\
\hline 9 & T2DG0901244 & 10 & T2DG1000566 & 6 & unrelated & 0 & 8 & half-first cousin & 0.112 \\
\hline 8 & T2DG0800497 & 9 & T2DG0901244 & 6 & unrelated & 0.007 & 8 & half-first cousin & 0.673 \\
\hline 21 & T2DG2100951 & 25 & T2DG2501033 & 6 & unrelated & 0 & 5 & first cousin & 0.633 \\
\hline 4 & T2DG0400207 & 4 & T2DG0400247 & 6 & unrelated & 0 & 5 & first cousin & 0.712 \\
\hline
\end{tabular}

Empirical $p$-values are based on 25,000 simulated replicates, with genotype data simulated under a specified relationship type. The simulating relationship type can be the null relationship defined by the given pedigrees (i.e. the null reltype) or another relationship type (i.e. the plausible reltype) as listed in Table 1 . The possible plausible relationship types are not unique and the table provides the one with the highest $p$-values. Small $p$-value for testing the null reltype suggests that the observed genotype data are not compatible with the null relationship defined by the given pedigree, whereas large $p$-value for testing the plausible reltype suggests that the observed genotype data are compatible with the proposed alternative.

efficiency. Although PREST-plus is more powerful than PLINK, there is a trade-off between statistical power and computational efficiency [5,6]. For large data sets involving analyzing millions of pairs, PLINK could be used as a screening tool for further analysis with PREST-plus.

Both PREST-plus and PLINK are sensitive to misspecified allele frequencies, therefore sensitive to population stratification and population admixture, in contrast to some recent work [eg, [18]]. However, results from other GAW18 study groups suggest that population admixture is not a major concern here. Nevertheless, robust relationship estimation and testing methods warrant further research.

The methods considered here focus on global estimation of IBD distribution, which is powerful and efficient to distinguish distinct relationships, for example, full-sibs versus unrelated. However, such global methods are not adequate to distinguish similar relationship types, for example, second-cousin versus unrelated. To this end, the recent local estimation methods [eg, [19]] provide useful research direction.

\section{Conclusions}

Pedigree errors and cryptic relatedness often occur in sample despite the best practice in data collection. Genome-wide marker data, collected for linkage or association studies, can provide accurate genealogy information between individuals. Using the GWAS SNP data, PREST-plus analyses the GAW18 sample that had been previously "cleaned," and it identifies 7 clearly misspecified relative pairs in the 20 SAFS families and 4 cryptic-related pairs in the set of putatively unrelated individuals.

\section{Competing interests}

The authors declare that they have no competing interests.

\section{Authors' contributions}

Study design: LS. Analysis: AD and LS. Manuscript drafting: LS and AD. All authors read and approved the final manuscript.

\section{Acknowledgements}

Genetic Analysis Workshop is supported by NIH grant R01 GM031575. The research of LS is supported by the Natural Sciences and Engineering Research Council of Canada (NSERC) and the Canadian Institutes of Health Research $(\mathrm{ClHR})$. 
The GAW18 whole genome sequence data were provided by the T2DGENES Consortium, which is supported by NIH grants U01 DK085524, U01 DK085584, U01 DK085501, U01 DK085526, and U01 DK085545. The other genetic and phenotypic data for GAW18 were provided by the San Antonio Family Heart Study and San Antonio Family Diabetes/Gallbladder Study, which are supported by NIH grants P01 HL045222, R01 DK047482, and R01 DK053889. The Genetic Analysis Workshop is supported by NIH grant R01 GM031575.

This article has been published as part of BMC Proceedings Volume 8 Supplement 1, 2014: Genetic Analysis Workshop 18. The full contents of the supplement are available online at http://www.biomedcentral.com/bmcproc/ supplements/8/S1. Publication charges for this supplement were funded by the Texas Biomedical Research Institute.

\section{Authors' details}

'Division of Biostatistics, Dalla Lana School of Public Health, University of Toronto, Canada. ${ }^{2}$ Department of Statistical Sciences, University of Toronto, Canada. ${ }^{3}$ Department of Clinical Biochemistry, University Health Network, Canada.

Published: 17 June 2014

\section{References}

1. Boehnke M, Cox NJ: Accurate inference of relationships in sib-pair linkage studies. Am J Hum Genet 1997, 61:423-429.

2. Voight BF, Pritchard JK: Confounding from cryptic relatedness in casecontrol association studies. PLoS Genet 2005, 1:e32.

3. Thornton T, McPeek MS: ROADTRIPS: case-control association testing with partially or completely unknown population and pedigree structure. Am J Hum Genet 2010, 86:172-184.

4. Price AL, Zaitlen NA, Reich D, Patterson N: New approaches to population stratification in genome-wide association studies. Nat Rev Genet 2010, 11:459-463.

5. Sun L: Detecting pedigree relationship errors. In Statistical Human Genetics:Methods and Protocols.. Humana Press;Elston R, Satagopan J, Sun S. NewYork 2012:25-46.

6. Sun L, Dimitromanolakis A: Identifying cryptic relationships. In Statistical Human Genetics : Methods and Protocols. Humana Press; Elston R, Satagopan J, Sun S. NewYork 2012:47-58.

7. Goring HH, Ott J: Relationship estimation in affected sib pair analysis of late-onset diseases. Eur J Hum Genet 1997, 5:69-77.

8. O'Connell JR, Weeks DE: Pedcheck: a program for identification of genotype incompatibilities in linkage analysis. Am J Hum Genet 1998, 63:259-266.

9. Ehm M, Wagner M: A test statistic to detect errors in sib-pair relationships. Am J Hum Genet 1998, 62:181-188.

10. McPeek MS, Sun L: Statistical tests for detection of misspecified relationships by use of genome-screen data. Am J Hum Genet 2000, 66:1076-1094

11. Sun L, Wilder K, McPeek MS: Enhanced pedigree error detection. Hum Hered 2002, 54:99-110.

12. Abecasis GR, Cherny SS, Cookson WO, Cardon LR: Grr: graphical representation of relationship errors. Bioinformatics 2001, 17:742-743.

13. Sieberts SK, Wijsman EM, Thompson EA: Relationship inference from trios of individuals in the presence of typing error. Am J Hum Genet 2002, 70:170-180.

14. Purcell $S$, Neale B, Todd-Brown K, Thomas L, Ferreira MAR, Bender D, Maller J, Sklar P, de Bakker PIW, Daly MJ, et al: Plink: a tool set for wholegenome association and population-based linkage analyses. Am J Hum Genet 2007, 81:559-575.

15. Dimitromanolakis A, Paterson AD, Sun L: Accurate IBD inference identifies cryptic relatedness in 9 HapMap populations. Abstract no 1768 presented at the Annual meeting of the American Society of Human Genetics 2009.

16. Thompson EA: The estimation of pairwise relationships. Ann Hum Genet 1975, 39:173-188.

17. Thompson EA: Pedigree Analysis in Human Genetics. Baltimore, MD, The Johns Hopkins University Press; 1986.

18. Thoronto T, Tang H, Hoffmann TJ, Ochs-Balcom HM, Caan BJ, Risch N: Estimating kinship in admixed populations. Am J Hum Genet 2012, 91:122-138.
19. Browning SR, Browning BL: Identity by decent between distant relatives: detection and applications. Annu Rev Genet 2012, 46:617-633.

doi:10.1186/1753-6561-8-S1-S23

Cite this article as: Sun and Dimitromanolakis: PREST-plus identifies pedigree errors and cryptic relatedness in the GAW18 sample using genome-wide SNP data. BMC Proceedings 2014 8(Suppl 1):S23.

\section{Submit your next manuscript to BioMed Central and take full advantage of:}

- Convenient online submission

- Thorough peer review

- No space constraints or color figure charges

- Immediate publication on acceptance

- Inclusion in PubMed, CAS, Scopus and Google Scholar

- Research which is freely available for redistribution

Submit your manuscript a www.biomedcentral.com/submit
C Biomed Central 